\title{
ISOTOPIC COMPOSITION OF LIGHT NUCLEI IN COSMIC RAYS: RESULTS FROM AMS-01
}

M. Aguilar ${ }^{1}$, J. Alcaraz ${ }^{1}$, J. Allaby ${ }^{2,37}$, B. Alpat ${ }^{3}$, G. Ambrosi ${ }^{3}$, H. Anderhub ${ }^{4}$, L. Ao ${ }^{5}$, A. Arefiev ${ }^{6}$, L. Arruda ${ }^{7}$, P. Azzarello ${ }^{3}$, M. Basile ${ }^{8}$, F. Barao ${ }^{7,9}$, G. Barreira ${ }^{7}$, A. Bartoloni ${ }^{10}$, R. Battiston ${ }^{3,11}$, R. BeCKer ${ }^{12}$, U. BeCKer ${ }^{12}$, L. Bellagamba ${ }^{8}$, J. Berdugo ${ }^{1}$, P. Berges ${ }^{12}$, B. Bertucci ${ }^{3}, 11$, A. Biland ${ }^{4}$, V. Bindi ${ }^{8}$, G. Boella ${ }^{13}$, M. Boschini ${ }^{13}$, M. Bourquin ${ }^{14}$, G. Bruni ${ }^{8}$, M. Buénerd ${ }^{15}$, J. D. Burger ${ }^{12}$, W. J. Burger ${ }^{11}$, X. D. CAi ${ }^{12}$, P. CANNARsa ${ }^{4}$, M. CAPEll ${ }^{12}$,

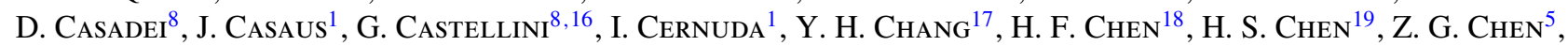

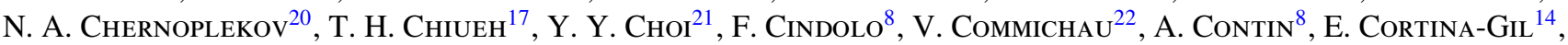
D. Crespo ${ }^{1}$, M. Cristinziani ${ }^{14}$, T. S. Dai ${ }^{12}$, C. Dela Guia ${ }^{1}$, C. Delgado ${ }^{1}$, S. Di Falco ${ }^{23}$, L. Djambazov ${ }^{4}$, I. D' Antone ${ }^{8}$,

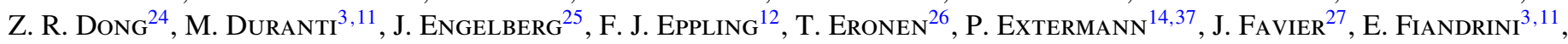

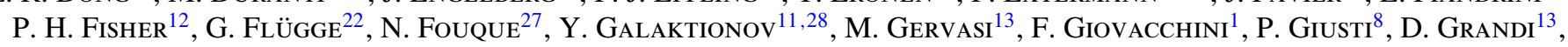

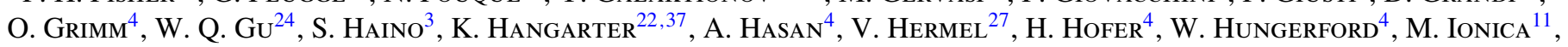
M. Jongmanns ${ }^{4}$, K. KarlamaA ${ }^{25}$, W. Karpinski ${ }^{28}$, G. $\mathrm{KenneY}^{4}$, D. H. Kim ${ }^{29}$, G. N. KIm ${ }^{29}$, K. S. Kim ${ }^{21}$, T. KIRN ${ }^{28}$, A. $\mathrm{KLimentov}^{6,12}$, R. KossaKowski ${ }^{27}$, A. Kounine ${ }^{12}$, V. Koutsenko ${ }^{6,12}$, M. Kraeber ${ }^{4}$, G. Laborie ${ }^{15}$, T. Laitinen $^{26}$, G. Lamanna ${ }^{27}$, G. Laurenti ${ }^{8}$, A. Lebedev ${ }^{12}$, C. Lechanoine-Leluc ${ }^{14}$, M. W. LeE ${ }^{29}$, S. C. LeE ${ }^{30}$, G. Levi ${ }^{8}$, C. H. Lin ${ }^{30}$,

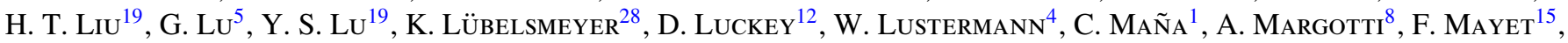

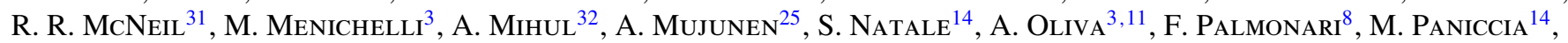
H. B. Park ${ }^{29}$, W. H. Park ${ }^{29}$, M. Pauluzzi ${ }^{3,11}$, F. Pauss ${ }^{4}$, R. Pereira ${ }^{7}$, E. Perrin ${ }^{14}$, A. Peviner ${ }^{33}$, F. Pilo ${ }^{23}$, M. Pimenta ${ }^{7}$,

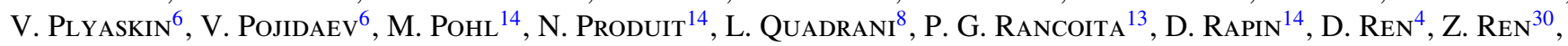

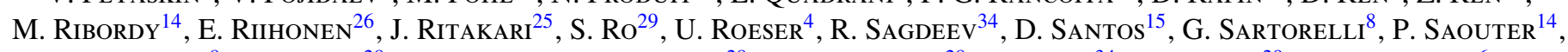
C. Sbarra ${ }^{8}$, S. Schael ${ }^{28}$, A. Schultz von Dratzig ${ }^{28}$, G. SchWering ${ }^{28}$, E. S. Seo ${ }^{34}$, J. W. Shin ${ }^{29}$, E. Shoumilov ${ }^{6}$, V. Shoutko ${ }^{12}$, T. Siedendurg ${ }^{12}$, R. Siedling ${ }^{28}$, D. Son ${ }^{29}$, T. Song ${ }^{24}$, F. R. SPAdA ${ }^{10}$, F. Sinella ${ }^{23}$, M. Steuer ${ }^{12}$, G. S. Sun $^{24}$, H. Suter ${ }^{4}$, X. W. Tang ${ }^{19}$, Samuel C. C. Ting ${ }^{12}$, S. M. Ting ${ }^{12}$, N. Tomassetti ${ }^{3}, 11$, M. Tornikoski ${ }^{25}$, J. Torsti ${ }^{26}$, J. TrümPer $^{35}$, J. Ulbricht ${ }^{4}$, S. UrPo ${ }^{25}$, E. Valtonen ${ }^{26}$, J. Vandenhirtz ${ }^{28}$, E. Velikhov ${ }^{20}$, B. Verlaat ${ }^{4}, 38$, I. Vetlitsky ${ }^{6}$, F. Vezzu ${ }^{15}$, J. P. Vialle ${ }^{27}$, G. Viertel ${ }^{4}$, D. Vité ${ }^{14}$, H. Von Gunten ${ }^{4}$, S. Waldmeier Wicki ${ }^{4}$, W. WallrafF ${ }^{28}$, J. Z. Wang ${ }^{5}$, K. Wiik ${ }^{25}$, C. Williams ${ }^{8}$, S. X. Wu ${ }^{12,17}$, P. C. XIA ${ }^{24}$, S. Xu ${ }^{12}$, Z. Z. Xu ${ }^{18}$, J. L. YAN ${ }^{5,37}$, L. G. YAN ${ }^{24}$, C. G. YANG ${ }^{19}$, J. YANG ${ }^{21}$, M. YANG ${ }^{19}$,

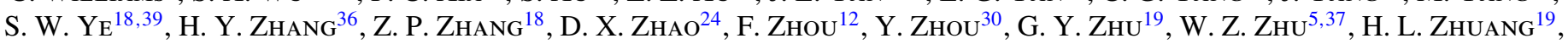
A. ZICHICHI ${ }^{8}$, B. ZIMMERMANN ${ }^{4}$, AND P. ZUCCON ${ }^{3}$

${ }^{1}$ Centro de Investigaciones Energéticas, Medioambientales y Tecnológicas, CIEMAT, E-28040 Madrid, Spain ${ }^{40}$

${ }^{2}$ European Laboratory for Particle Physics, CERN, CH-1211 Geneva 23, Switzerland ${ }^{3}$ INFN-Sezione di Perugia, I-06100 Perugia, Italy ${ }^{41}$

${ }_{5}^{4}$ Eidgenössische Technische Hochschule, ETH Zürich, CH-8093 Zürich, Switzerland

${ }^{5}$ Chinese Academy of Launching Vehicle Technology, CALT, 100076 Beijing, China

${ }^{6}$ Institute of Theoretical and Experimental Physics, ITEP, Moscow 117259, Russia

${ }^{7}$ Laboratorio de Instrumentacao e Fisica Experimental de Particulas, LIP, P-1000 Lisboa, Portugal

${ }^{8}$ Dipartimento di Fisica and INFN, Università di Bologna, I-40126 Bologna, Italy ${ }^{41}$

${ }^{9}$ Instituto Superior Técnico, IST, P-1096 Lisboa, Portugal ${ }^{10}$ INFN-Sezione di Roma, I-00185 Roma, Italy ${ }^{41}$

${ }^{11}$ Dipartimento di Fisica, Università Degli Studi di Perugia, I-06100 Perugia, Italy; Nicola.Tomassetti@pg.infn.it

${ }^{12}$ Massachusetts Institute of Technology, Cambridge, MA 02139, USA

${ }^{13}$ INFN-Sezione di Milano, I-20133 Milan, Italy ${ }^{41}$

${ }^{14}$ DPNC, Université de Genève, CH-1211 Geneva 4, Switzerland

${ }^{15}$ LPSC, Université Joseph Fourier Grenoble 1, CNRS/IN2P3, Institut Polytechnique de Grenoble, F-38026 Grenoble, France ${ }^{16}$ CNR-IROE, I-50125 Florence, Italy

${ }^{17}$ Department of Physics, National Central University, Chung-Li 32054, Taiwan

${ }^{18}$ Chinese University of Science and Technology, USTC, Hefei, Anhui 230 029, China ${ }^{42}$

${ }^{19}$ Institute of High Energy Physics, IHEP, Chinese Academy of Sciences, 100039 Beijing, China ${ }^{42}$

${ }^{20}$ Kurchatov Institute, Moscow 123182, Russia

${ }^{21}$ Department of Physics, Ewha Womens University, 120-750 Seoul, Republic of Korea

${ }^{22}$ III. Physikalisches Institut, RWTH, D-52074 Aachen, Germany ${ }^{43}$

${ }^{23}$ Dipartimento di Fisica and INFN, Università di Pisa, I-56100 Pisa, Italy ${ }^{41}$

${ }^{24}$ Institute of Electrical Engineering, IEE, Chinese Academy of Sciences, 100080 Beijing, China

${ }^{25}$ Helsinki University of Technology, FIN-02540 Kylmala, Finland

${ }^{26}$ Space Research Laboratory (SRL), University of Turku, FIN-20014 Turku, Finland

${ }^{27}$ LAPP, Université de Savoie, CNRS/IN2P3, F-74941 Annecy-le-Vieux, France ${ }^{28}$ I. Physikalisches Institut, RWTH, D-52074 Aachen, Germany ${ }^{43}$

${ }^{29}$ CHEP, Kyungpook National University, 702-701 Daegu, Republic of Korea

${ }^{30}$ Institute of Physics, Academia Sinica, Nankang Taipei 11529, Taiwan

${ }^{31}$ Department of Physics and Astronomy, Louisiana State University, Baton Rouge, LA 70803, USA

${ }^{32}$ Institute of Microtechnology, Politechnica University of Bucharest and University of Bucharest, R-76900 Bucharest, Romania

${ }^{33}$ Department of Physics and Astronomy, Johns Hopkins University, Baltimore, MD 21218, USA

${ }^{34}$ Institute for Physical Science and Technology, University of Maryland, College Park, MD 20742, USA

${ }_{35}$ Max-Planck-Institut für extraterrestrische Physik, D-85740 Garching, Germany

${ }^{36}$ Center of Space Science and Application, Chinese Academy of Sciences, 100080 Beijing, China Received 2010 December 22; accepted 2011 May 10; published 2011 July 13 


\section{ABSTRACT}

The variety of isotopes in cosmic rays allows us to study different aspects of the processes that cosmic rays undergo between the time they are produced and the time of their arrival in the heliosphere. In this paper, we present measurements of the isotopic ratios ${ }^{2} \mathrm{H} /{ }^{4} \mathrm{He},{ }^{3} \mathrm{He} /{ }^{4} \mathrm{He},{ }^{6} \mathrm{Li} /{ }^{7} \mathrm{Li},{ }^{7} \mathrm{Be} /\left({ }^{9} \mathrm{Be}+{ }^{10} \mathrm{Be}\right)$, and ${ }^{10} \mathrm{~B} /{ }^{11} \mathrm{~B}$ in the range $0.2-1.4 \mathrm{GeV}$ of kinetic energy per nucleon. The measurements are based on the data collected by the Alpha Magnetic Spectrometer, AMS-01, during the STS-91 flight in 1998 June.

Key words: acceleration of particles - cosmic rays - nuclear reactions, nucleosynthesis, abundances

\section{INTRODUCTION}

Cosmic rays (CRs) detected with kinetic energies in the range from $\mathrm{MeV}$ to $\mathrm{TeV}$ per nucleon are believed to be produced by galactic sources. Observations of X-ray and $\gamma$-ray emission from galactic sites such as supernova remnants, pulsars, or stellar winds reveal the presence of energetic particle acceleration mechanisms occurring in such objects. The subsequent destruction of these accelerated nuclei (e.g., p, $\mathrm{He}, \mathrm{C}, \mathrm{N}, \mathrm{O}, \mathrm{Fe}$ ) in the interstellar medium gives rise to secondary species that are rare in the $\mathrm{CR}$ sources, such as $\mathrm{Li}, \mathrm{Be}, \mathrm{B}$, sub-Fe elements, deuterons, antiprotons, positrons, and high energy photons. The relation between secondary CRs and their primary progenitors allows the determination of propagation parameters such as the diffusion coefficient and the size of the diffusion region. For a recent review, see Strong et al. (2007).

Along with the ratios $\mathrm{B} / \mathrm{C}$ and sub-Fe/Fe, it is of great importance to determine the propagation history of the lighter $\mathrm{H}, \mathrm{He}, \mathrm{Li}$, and $\mathrm{Be}$ isotopes. Since ${ }^{2} \mathrm{H}$ and ${ }^{3} \mathrm{He} \mathrm{CRs}$ are mainly produced by the breakup of the primary ${ }^{4} \mathrm{He}$ in the galaxy, the ratios ${ }^{2} \mathrm{H} /{ }^{4} \mathrm{He}$ and ${ }^{3} \mathrm{He} /{ }^{4} \mathrm{He}$ probe the propagation history of helium (Webber 1997). The isotopes of $\mathrm{Li}, \mathrm{Be}$, and $\mathrm{B}$, all of secondary origin, are also useful for a quantitative understanding of $\mathrm{CR}$ propagation. The relative abundances and isotopic composition of $\mathrm{H}, \mathrm{He}, \mathrm{Li}, \mathrm{Be}$, and $\mathrm{B}$, therefore, might help to distinguish between the propagation models and give constraints on their parameters (Moskalenko et al. 2003).

Low energy data $\left(\lesssim 200 \mathrm{MeV}\right.$ nucleon $\left.^{-1}\right)$ on CR isotopic composition come mainly from space experiments such as the HET telescopes on Voyager 1 and 2 (Webber et al. 2002), the Cosmic Ray Isotope Spectrometer (CRIS) on the Advanced Composition Explorer (ACE) satellite (de Nolfo et al. 2001), the Ulysses high energy telescope (Connell 1998), the Interplanetary Monitoring Platform (IMP; Garcia-Munoz 1977), and the HKH experiment on the International Sun-Earth Explorer (ISEE) spacecraft (Wiedenbeck \& Greiner 1980). Light nuclei data at higher energies (up to a few $\mathrm{GeV}$ nucleon ${ }^{-1}$ ) have been measured by balloon-borne magnetic spectrometers including IMAX (Reimer et al. 1998), ISOMAX (Hams et al. 2004), SMILI (Ahlen et al. 2000), BESS (Wang et al. 2002), and the Goddard Space Flight Center (GSFC) balloon (Hagen et al. 1977).

AMS-01 observed CRs at an altitude of $\sim 380 \mathrm{~km}$ during a period, 1998 June, of relatively quiet solar activity. It collected data free from atmospheric induced background. In this paper,

\footnotetext{
${ }^{37}$ Deceased.

${ }^{38}$ Now at National Institute for High Energy Physics, NIKHEF, NL-1009 DB Amsterdam, the Netherlands.

${ }^{39}$ Supported by ETH Zürich.

${ }^{40}$ Also supported by the Comisión Interministerial de Ciencia y Tecnología.

41 Also supported by the Italian Space Agency.

42 Supported by the National Natural Science Foundation of China.

${ }^{43}$ Supported by the Deutsches Zentrum für Luft- und Raumfahrt, DLR.
}

we present measurements of the ${ }^{3} \mathrm{He} /{ }^{4} \mathrm{He}$ ratio over the kinetic energy range $0.2-1.4 \mathrm{GeV}$ nucleon ${ }^{-1}$, and the average values of the ratios ${ }^{6} \mathrm{Li} /{ }^{7} \mathrm{Li},{ }^{7} \mathrm{Be} /\left({ }^{9} \mathrm{Be}+{ }^{10} \mathrm{Be}\right)$, and ${ }^{10} \mathrm{~B} /{ }^{11} \mathrm{~B}$ over the same energy range. The ratio ${ }^{2} \mathrm{H} /{ }^{4} \mathrm{He}$ is also presented.

\section{THE ALPHA MAGNETIC SPECTROMETER}

The Alpha Magnetic Spectrometer (AMS) is a particle physics instrument designed for the high-precision and longduration measurement of CRs in space. The AMS-01 precursor experiment operated successfully during a 10 day flight on the space shuttle Discovery (STS-91).

The spectrometer was composed of a cylindrical permanent magnet, a silicon micro-strip tracker, time-of-flight (TOF) scintillator planes, an aerogel Čerenkov counter, and anticoincidence counters. The performance of AMS-01 is described elsewhere (Aguilar et al. 2002).

Data collection started on 1998 June 3. The orbital inclination was 51.7 and the geodetic altitude ranged from 320 to $390 \mathrm{~km}$. The data were collected in four phases: (1) 1 day of check out before docking with the Mir space station, (2) 4 days while docked to Mir, (3) 3.5 days with AMS pointing directions within $0^{\circ}, 20^{\circ}$, and $45^{\circ}$ of the zenith, and (4) 0.5 days before descending, pointing toward the nadir.

The acceptance criterion of the trigger logic in the AMS-01 instrument was a four-fold coincidence between the signals from the four TOF planes. Only particles traversing the silicon tracker were accepted. Events crossing the anti-coincidence counters or producing multiple hits in the TOF layers were rejected. A prescaled subsample of 1 out of 1000 events was recorded with a dedicated minimum-bias configuration. This "unbiased trigger" required only the TOF coincidence.

The AMS-01 mission provided results on CR protons, helium, electrons, positrons, and light nuclei (Aguilar et al. 2002, 2007, 2010). During the flight, a total of 99 million triggers were recorded by the spectrometer, with 2.85 million helium nuclei and nearly 200,000 nuclei with charge $Z>2$.

\section{DATA ANALYSIS}

The identification of CR nuclei with AMS-01 was performed through the combination of independent measurements provided by the various detectors. The particle rigidity, $R$ (momentum per unit charge, $p c / Z e$ ), was provided by the deflection of the reconstructed particle trajectory in the magnetic field. The velocity, $\beta=v / c$, was measured from the particle transit time between the four TOF planes along the track length. The reconstruction algorithm provided, together with the measured quantities $R$ and $\beta$, an estimation of their uncertainties $\delta R$ (from tracking) and $\delta \beta$ (from timing) that reflected the quality of the spectrometer in performing such measurements. The particle charge magnitude $|Z|$ was obtained by the analysis of the multiple measurements of energy deposition in the four TOF 
scintillators up to $Z=2$ (Aguilar et al. 2002) and the six silicon layers up to $Z=8$ (Aguilar et al. 2010). The particle mass number, $A$, was therefore determined from the resulting charge, velocity, and rigidity:

$$
A=\frac{R Z e}{m_{n} \beta c^{2}} \sqrt{1-\beta^{2}},
$$

where $m_{n}$ is the nucleon mass.

The response of the detector was simulated using the AMS simulation program, based on GEANT-3.21 (Brun et al. 1987) and interfaced with the hadronic package RQMD (relativistic quantum molecular dynamics; Sorge 1995). The effects of energy loss, multiple scattering, nuclear interactions, and decays were included, as well as detector efficiency and resolution. After the flight, the detector was extensively calibrated at GSI, Darmstadt, with ion beams (He, C) and at the CERN Proton Synchrotron (CERN-PS), Geneva, with proton beams. This ensured that the performance of the detector and the analysis procedure were thoroughly understood.

Further details are found in Aguilar et al. (2002) and references therein.

\subsection{Helium Isotopes}

Given the large amount of statistics available for $Z=2$ data, we considered only the highest quality data collected during the post-docking phase (3) and only while pointing toward the zenith. Data taken while passing near the South Atlantic Anomaly (latitude: $5^{\circ}-45^{\circ} \mathrm{S}$; longitude: $5^{\circ}-85^{\circ} \mathrm{W}$ ) were excluded. Only events taken when the energy interval $0.2-1.4 \mathrm{GeV}$ nucleon ${ }^{-1}$ was above the geomagnetic cutoff for both the isotopes ${ }^{3} \mathrm{He}$ and ${ }^{4} \mathrm{He}$ were kept; this corresponds to selecting the orbital regions with the highest geomagnetic latitudes, $\Theta_{M}$, roughly $\left|\Theta_{M}\right| \gtrsim 0.9$.

Furthermore, the acceptance was restricted to particles traversing the detector top-down within $30^{\circ}$ of the positive $z$-axis. Events with poorly reconstructed trajectories were rejected through quality cuts on the associated $\chi^{2}$ or consistency requirements between the two reconstructed half-tracks (Aguilar et al. 2010). To avoid biasing the reconstructed mass distributions, no cuts on the consistency of the TOF velocity versus tracker rigidity measurements were applied. We required that the velocity was measured with hits from at least three out of four TOF planes and that the rigidity was reconstructed with at least five out of six tracker layers. Approximately 18,000 nuclei with charge $Z=2$ were selected in the energy range 0.2-1.4 GeV nucleon ${ }^{-1}$. The charge was determined from the energy depositions in both the TOF and tracker layers. The kinetic energy per nucleon was measured with the TOF system, i.e., through the velocity $\beta$. In the energy range considered, the TOF energy resolution is comparable to that of the tracker.

The selected data are shown in Figure 1 distributed in the $(\beta, R)$ plane. The two curves represent the exact relation between velocity $\beta$ and rigidity $R$ for a $Z=2$ nucleus of mass number $A=3$ (dashed line) and $A=4$ (solid line), which is

$$
\beta=\left[1+A^{2}\left(\frac{m_{n} c^{2}}{Z e R}\right)^{2}\right]^{-1 / 2}
$$

The large dispersion of the measured data, apparent from Figure 1, indicates a relatively poor mass resolution in the separation of the two mass numbers. Under these conditions, any event-to-event separation (e.g., through a mass cut) is clearly

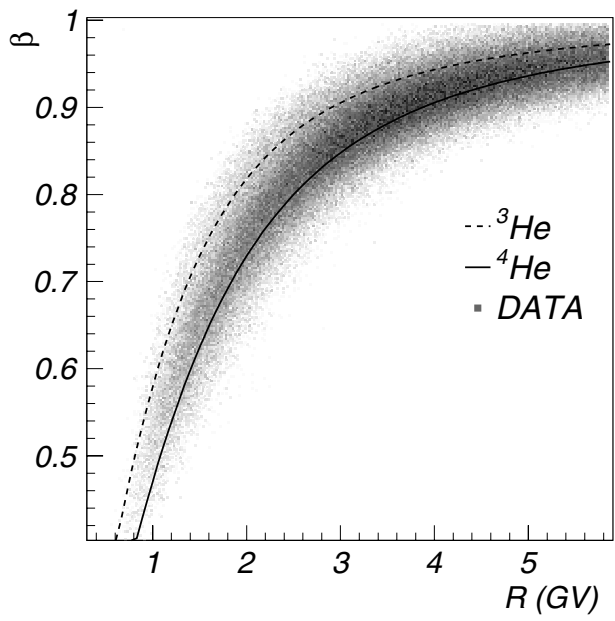

Figure 1. Distribution of the measured velocity, $\beta$, as a function of the reconstructed rigidity, $R$, for $Z=2$ nuclei. The two lines represent the exact relationship of $\beta$ to $R$ for the two isotopes ${ }^{3} \mathrm{He}$ (dashed line) and ${ }^{4} \mathrm{He}$ (solid line).

inapplicable. In addition, the distribution of the reconstructed mass numbers (Equation (1)) exhibits asymmetric tails; so the standard Gaussian fit method (Seo et al. 1997) is not appropriate for describing the observed mass response of the instrument.

In order to determine the isotopic ratios, it was therefore necessary to develop a comprehensive model for the complete response of the instrument to different masses. The mass resolution is influenced by the intrinsic time resolution of the TOF system and by the bending power of the magnet coupled with the intrinsic spatial resolution of the tracker. Physical processes such as multiple scattering, energy losses, and interactions along the particle path also contribute in shaping the reconstructed mass distributions. Thus, we modeled the AMS-01 mass response by means of our Monte Carlo (MC) simulation program, which includes all the aforementioned physical effects as well as the instrumental readout, providing a realistic description of particle tracking and timing on an eventby-event basis. The program was also tuned with data collected during the test beams. The resulting rigidity resolution $\delta R / R$ and velocity resolution $\delta \beta / \beta$ are shown in Figure 2 for test beam data (filled circles) and MC events (histograms). It can be seen that the mass resolution, given approximately by

$$
\left(\frac{\delta A}{A}\right)^{2}=\left(\gamma^{2} \frac{\delta \beta}{\beta}\right)^{2}+\left(\frac{\delta R}{R}\right)^{2},
$$

was correctly simulated as the MC simulation agrees with the data within $\sim 2 \%$.

Using a sufficiently large number of simulated events of ${ }^{3} \mathrm{He}$ and ${ }^{4} \mathrm{He}$ and with the ratio ${ }^{3} \mathrm{He} /{ }^{4} \mathrm{He}$ of the detected events as a free parameter, we determined the best composition fit between the simulated mass distributions and the measured one. In these fits the overall normalization, $\mathcal{N}$, was also a free parameter. In principle, $\mathcal{N}$ should be fixed by the data, namely by the number of entries, $\mathcal{N}_{\mathrm{E}}$, of each mass histogram. Deviations of $\mathcal{N}$ from its expected value $\mathcal{N}_{\mathrm{E}}$ may indicate the presence of an unaccounted background, e.g., from charge misidentification.

The results of this procedure are shown in Figure 3, where the agreement between the measured mass histograms (filled circles) and the simulated histograms (lines) turned out to be very satisfactory. The fits of the ${ }^{3} \mathrm{He} /{ }^{4} \mathrm{He}$ mass composition ratios gave unique minima in all the energy bins considered. 

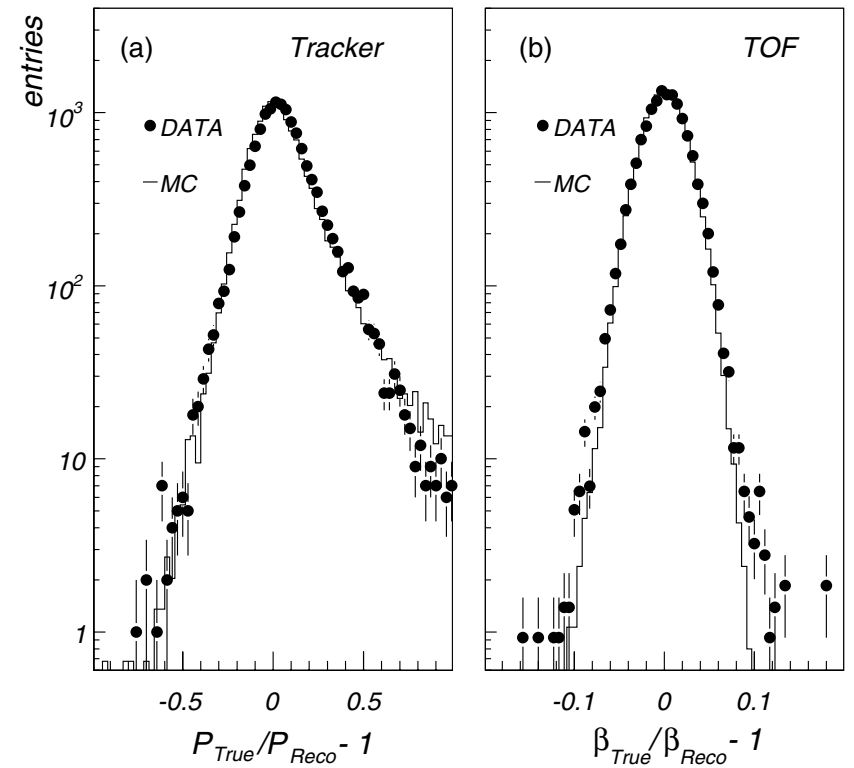

Figure 2. (a) Rigidity and (b) velocity resolutions of the AMS-01 tracker and TOF estimated with measured data from the test beam with $E=$ $2 \mathrm{GeV}$ nucleon ${ }^{-1}$ helium nuclei. Data (circles) are compared with the MC simulation (histograms). The MC entries are normalized to the data.

The uncertainties associated with these ratios were determined directly from the $1 \sigma$ uncertainties in the $\chi^{2}$ statistics of the fitting procedure. The $\chi^{2}$ fitting method was cross-checked with the maximum likelihood method. The two methods gave the
Table 1

Fit Results and Correction Factors for the Ratio ${ }^{3} \mathrm{He} /{ }^{4} \mathrm{He}$ Between 0.2 and $1.4 \mathrm{GeV}$ of Kinetic Energy per Nucleon

\begin{tabular}{lcccccc}
\hline \hline Energy & Events & Fit Results & $\chi^{2} / \mathrm{df}$ & $\delta A / A$ & ACorr & FCorr \\
\hline $0.20-0.30$ & 2,660 & $0.125 \pm 0.011$ & $32.3 / 29$ & $13.1 \%$ & 1.12 & 0.97 \\
$0.30-0.44$ & 3,553 & $0.158 \pm 0.096$ & $51.1 / 29$ & $12.2 \%$ & 1.05 & 0.98 \\
$0.44-0.64$ & 3,867 & $0.182 \pm 0.094$ & $65.8 / 34$ & $11.8 \%$ & 1.00 & 0.98 \\
$0.64-0.95$ & 4,142 & $0.211 \pm 0.098$ & $62.7 / 30$ & $12.2 \%$ & 0.99 & 0.98 \\
$0.95-1.40$ & 3,813 & $0.223 \pm 0.012$ & $55.0 / 33$ & $13.9 \%$ & 0.99 & 0.98 \\
\hline
\end{tabular}

same results and very similar uncertainties. Double Gaussian fits were also performed in order to provide the corresponding mass resolution, $\delta A / A$, for each energy bin, defined as the ratio between the width and the mean. ${ }^{44}$ The fitted ${ }^{3} \mathrm{He} /{ }^{4} \mathrm{He}$ ratios for all the energy bins considered from $200 \mathrm{MeV}$ nucleon $^{-1}$ to $1.4 \mathrm{GeV}$ nucleon ${ }^{-1}$ are listed in Table 1 , together with the $\chi^{2} / \mathrm{df}$ values, the number of events, the mass resolution, and correction factors discussed below.

\subsection{Top-Of-Instrument Corrections}

The measured mass distribution of Figure 3 was fitted with an MC sample of mixed ${ }^{3} \mathrm{He}$ and ${ }^{4} \mathrm{He}$ nuclei that were sent through the same analysis chain (trigger, reconstruction, and data selection) as the data. The free parameter is the ratio ${ }^{3} \mathrm{He} /$ ${ }^{4} \mathrm{He}$ of the two mass distributions corresponding to the recorded

$\overline{{ }^{44} \text { Equal mass resolutions were obtained for }}{ }^{3} \mathrm{He}$ and ${ }^{4} \mathrm{He}$ within $0.1 \%$ at all energies. Table 1 provides the mean values.
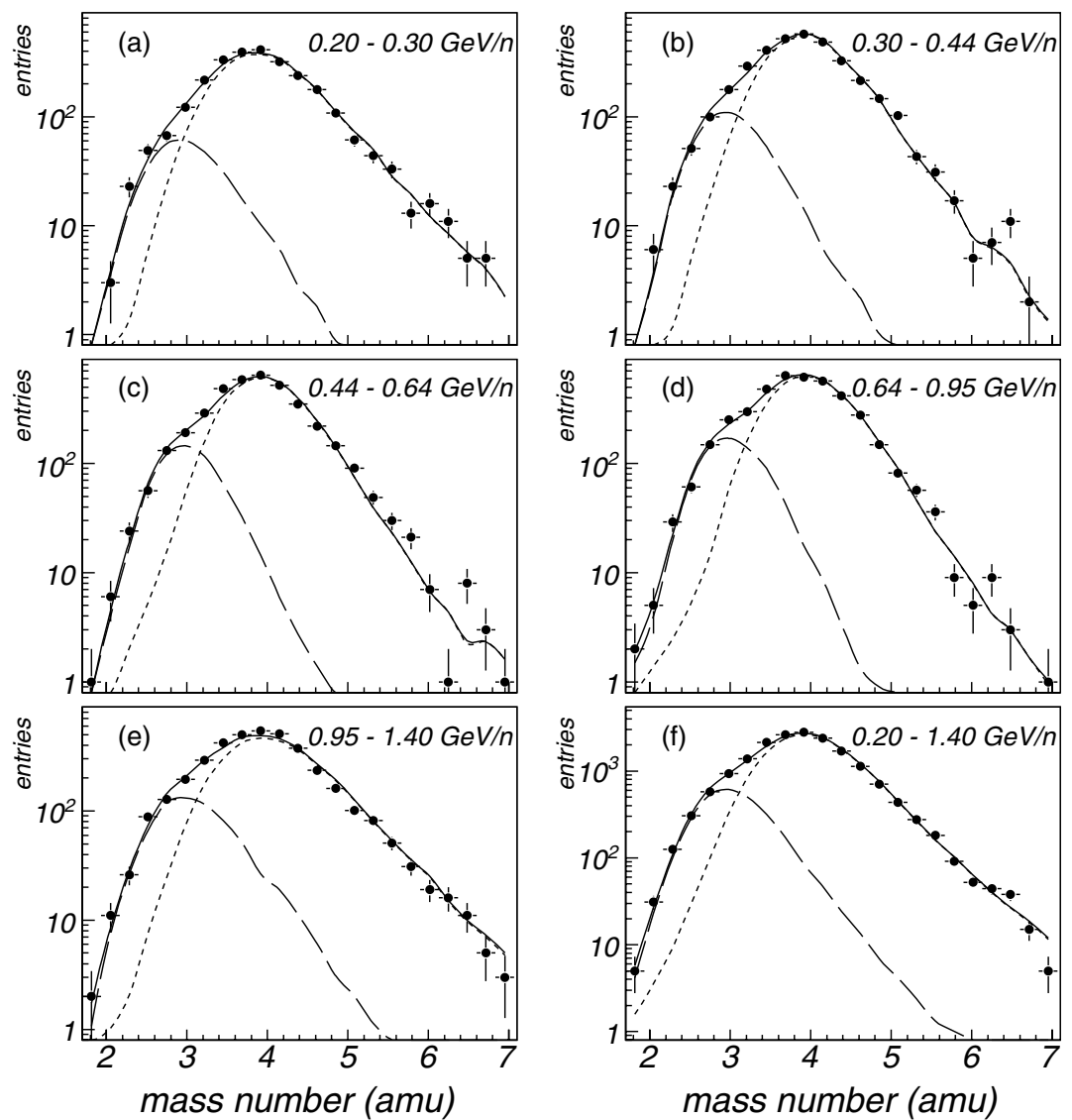

Figure 3. Reconstructed mass distributions of $Z_{\text {rec }}=2$ events from flight data (solid circles) and from MC generated isotopes of ${ }^{3} \mathrm{He}$ (long-dashed lines), ${ }^{4} \mathrm{He}$ (short-dashed lines), and their sum (solid lines). Distributions are shown in five energy intervals from (a) to (e) and over the entire range (f) between 0.2 and $1.4 \mathrm{GeV}$ nucleon $^{-1}$. 


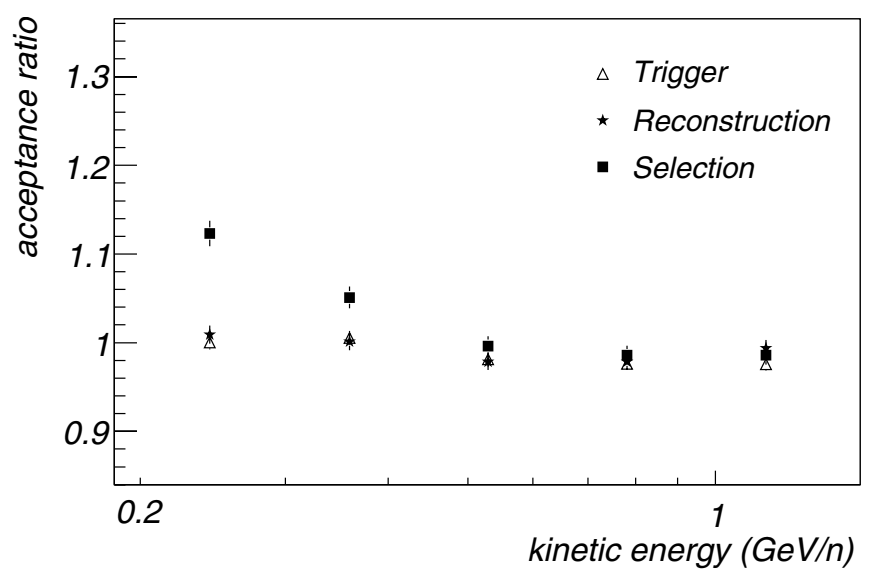

Figure 4. Ratios of the acceptances of ${ }^{4} \mathrm{He}$ and ${ }^{3} \mathrm{He}$ as a function of kinetic energy per nucleon. This quantity is shown after the successive application of the trigger (open triangles), reconstruction (stars), and selection (filled squares) cuts.

events. Hence, small corrections have to be performed in order to extract the ratio of interest, namely the ratio entering the instrument. These are referred to as Top-Of-Instrument (TOI) corrections.

\subsubsection{Acceptance Corrections}

The detector acceptance, $\mathcal{A}$, includes trigger efficiency, reconstruction efficiency, and selection efficiency. The acceptance was calculated using our MC simulation program. Nucleus trajectories were simulated in the energy range $\sim 0.05-40 \mathrm{GeV}$ nucleon ${ }^{-1}$. They were emitted downward from a square of length $3.9 \mathrm{~m}$ placed above the detector. In total, 110 million ${ }^{3} \mathrm{He}$ and 550 million ${ }^{4} \mathrm{He}$ nuclei were simulated. The physical processes involved and the detector response are very similar for the two isotopes, i.e., the resulting acceptances are quite similar in magnitude. The contributions from the detector acceptance mostly cancel in the ratio. The associated systematic errors also cancel. The only important factor in determining the isotopic ratios is the knowledge of any isotopic-dependent effects in the detector response. Mass-dependent features are expected from the following effects.

1. Rigidity threshold. The instrument acceptance is rigidity dependent, because the tracks of slower particles are more curved, and it is less likely that they pass through both the upper and lower TOF counters and the tracking volume. Since, at the same kinetic energy per nucleon, the lighter isotope ${ }^{3} \mathrm{He}$ has lower rigidity than the heavier ${ }^{4} \mathrm{He}$, the resulting acceptance, particularly at lower energies, is lower for ${ }^{3} \mathrm{He}$. Above $0.2 \mathrm{GeV}$ nucleon ${ }^{-1}$, the rigidity threshold affects the ratio by less than $\sim 1 \%$.

2. Multiple scattering. Coulomb scattering is slightly more pronounced for lighter particles. Since multiple scattering affects the event reconstruction and selection efficiency, the acceptance for the lighter isotope is smaller in the lowest energy region. The multiple scattering effect amounts to $\sim 10 \%$ at $E \sim 0.2 \mathrm{GeV}$ nucleon $^{-1}$ and decreases with energy, down to $\sim 1 \%$ at $E \sim 1 \mathrm{GeV}$ nucleon $^{-1}$.

3. Nuclear interactions. The attenuation of CRs after traversing the TOI material is isotope dependent and closely related to the inelastic cross sections, $\sigma_{\text {int }}$, for the interactions in the various layers of the detector material. For ${ }^{3} \mathrm{He}$ and ${ }^{4} \mathrm{He}$, the attenuation due to interactions differs by $\sim 2 \%$ or less.

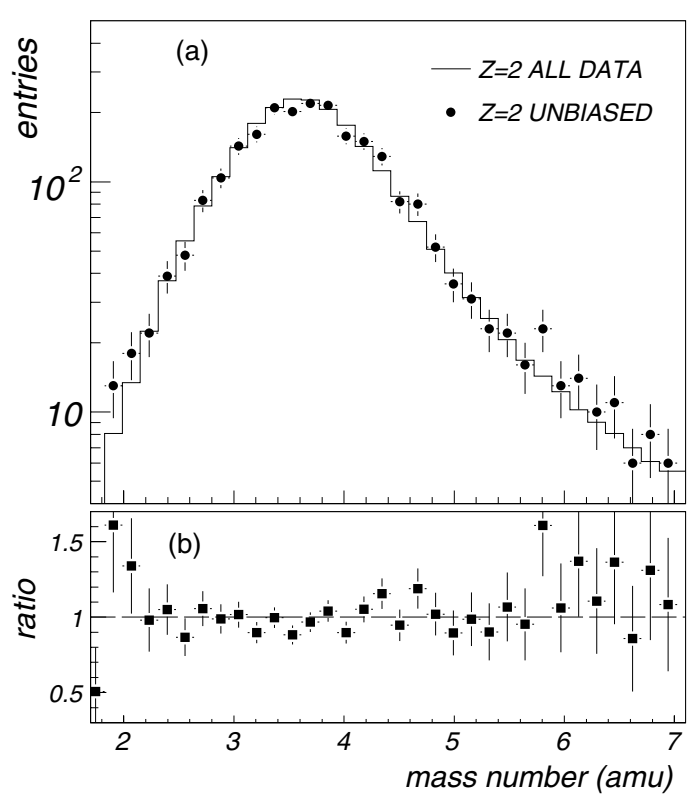

Figure 5. (a) Reconstructed mass distribution of $Z=2$ events collected with the unbiased trigger (filled circles) in comparison with the $Z=2$ mass histogram obtained with all the data (histogram). (b) Ratio of the two histograms (squares); the horizontal dashed line is shown for reference.

Table 2

Material Above the Tracker

\begin{tabular}{lcc}
\hline \hline Detector Element & Composition & Amount \\
\hline MLI thermal blanket & $\mathrm{C}_{5} \mathrm{H}_{4} \mathrm{O}_{2}$ & $0.7 \mathrm{~g} \mathrm{~cm}^{-2}$ \\
LEP shield & $\mathrm{C}$ & $1.3 \mathrm{~g} \mathrm{~cm}^{-2}$ \\
TOF scintillator & $\mathrm{C} / \mathrm{H}=1$ & $2.1 \mathrm{~g} \mathrm{~cm}^{-2}$ \\
TOF support structure & $\mathrm{Al}$ & $1.0 \mathrm{~g} \mathrm{~cm}^{-2}$ \\
\hline
\end{tabular}

Note. The column density is averaged over the angle of incidence.

Figure 4 shows the ratio of the two acceptances for MC events after the successive application of the trigger, reconstruction, and then selection cuts. Deviations are appreciable below $0.4 \mathrm{GeV}$ nucleon $^{-1}$ and mainly due to the event selection (filled squares). This indicates the dominance of the "multiple scattering effect" mentioned above, because the selection cuts acted against events with large scattering angles.

These mass-dependent features, due to the particle dynamics in the detector, did not appreciably influence the AMS-01 trigger system. In Figure 5, we report the reconstructed mass distribution using data collected with the unbiased trigger (Section 2). The comparison of such unbiased data (circles) with all $Z=2$ data collected from the flight (solid line, normalized to the unbiased data entries) shows no significant difference in the mass distribution.

\subsubsection{Nuclear Interactions}

Table 2 lists the material between the top of the payload and the tracker: a multi-layer insulation (MLI) blanket, a low energy particle (LEP) shield, and two TOF layers of plastic scintillators supported by a honeycomb structure. In total there were $\sim 5 \mathrm{~g} \mathrm{~cm}^{-2}$ of material above the tracking volume.

The RQMD interface used in the AMS simulation program provided a simulation of all the high energy hadronic collisions involving deuterons, ${ }^{3} \mathrm{He},{ }^{4} \mathrm{He}$, and heavier ions. These effects give an appreciable contribution to the total acceptance of Section 3.2.1. The survival probability of ${ }^{3} \mathrm{He}$ 
$\left({ }^{4} \mathrm{He}\right)$ after traversing the TOI material of Table 2 varies between $\sim 90 \%(\sim 88 \%)$ at $\sim 0.2 \mathrm{GeV}$ nucleon $^{-1}$ and $\sim 86 \%(\sim 86 \%)$ at $\sim 1.4 \mathrm{GeV}$ nucleon $^{-1}$.

More critical is the fragmentation of ${ }^{4} \mathrm{He}$ into ${ }^{3} \mathrm{He}$ that required a dedicated correction. In this process, if only a neutron is "stripped" above the tracker, the event is recorded as a clean ${ }^{3} \mathrm{He}$ event. The measured ${ }^{3} \mathrm{He} /{ }^{4} \mathrm{He}$ ratio is then distorted by incoming ${ }^{4} \mathrm{He}$ that spill over into the ${ }^{3} \mathrm{He}$ mass distribution. Note that the simulated mass distributions of Figure 3 are referred to the particle identities within the tracking volume, i.e., the ${ }^{3} \mathrm{He}$ mass histograms of the figures (long-dashed lines) also contain the "extra" ${ }^{3} \mathrm{He}$ nuclei generated as fragmentation products of ${ }^{4} \mathrm{He}$. Assuming that the kinetic energy per nucleon is maintained in the mass changing process, the ratio has been corrected for this effect. For each energy interval considered, the ratio $\eta$ between the "extra" ${ }^{3} \mathrm{He}$ and the total number of detected ${ }^{4} \mathrm{He}$ was estimated. The isotopic ratio ${ }^{3} \mathrm{He} /{ }^{4} \mathrm{He}$ resulting from the composition fit $\mathcal{M}$ is then related to the TOI ratio $\mathcal{R}$ through the relation

$$
\mathcal{M}=\frac{\mathcal{A}_{3} \phi_{3}+\eta \mathcal{A}_{4} \phi_{4}}{\mathcal{A}_{4} \phi_{4}-\eta \mathcal{A}_{4} \phi_{4}}=\left(\frac{1}{1-\eta}\right)\left[\left(\frac{\mathcal{A}_{3}}{\mathcal{A}_{4}}\right) \mathcal{R}+\eta\right],
$$

where $\phi_{3}$ and $\phi_{4}$ are the incident (TOI) intensities of the two isotopes ( $\left.\mathcal{R} \equiv \phi_{3} / \phi_{4}\right)$ and $\mathcal{A}_{3}$ and $\mathcal{A}_{4}$ the corresponding acceptances. Inverting Equation (4), we obtain the TOI ratio:

$$
\mathcal{R}=\left(\frac{\mathcal{A}_{4}}{\mathcal{A}_{3}}\right)\left[1-\eta-\frac{\eta}{\mathcal{M}}\right] \mathcal{M} .
$$

We then define the TOI correction factors as ACorr $\equiv \mathcal{A}_{4} / \mathcal{A}_{3}$ for the acceptance and FCorr $\equiv 1-\eta-\frac{\eta}{\mathcal{M}}$ for the fragmentation. Note that ACorr is the quantity shown in Figure 4 (filled squares). These values, to be applied as multiplicative factors to the fitted ratio, are also listed in Table 1. Such corrections are affected by $\lesssim 3 \%$ uncertainties in total, associated with the various physical and instrumental effects discussed here and in Section 3.2.1. All these errors and their role in the ${ }^{3} \mathrm{He} /{ }^{4} \mathrm{He}$ ratio are reviewed in Section 3.3.

\subsection{3. $\delta$-Ray Emission}

The effect of $\delta$-rays was included in our MC simulation. In our previous work (Aguilar et al. 2010), it was noted that energetic knock-on electrons affect the total acceptance at high energies. The production of $\delta$-rays is proportional to the square of the primary particle charge, and the maximum energy of the $\delta$-rays produced, $E_{\max }^{\delta}$, is proportional to the primary particle energy; for a nucleus of momentum $M \gamma \beta c$, approximately

$$
E_{\max }^{\delta}=2 m_{e} c^{2} \beta^{2} \gamma^{2}
$$

For high energy nuclei $\left(E \gtrsim \mathrm{GeV}\right.$ nucleon $^{-1}$ ), the emitted electrons can reach the anti-coincidence counters and veto the event, leading to an energy- and charge-dependent trigger efficiency. At lower energy, the $\delta$-rays curl up inside the tracking volume, affecting the reconstruction efficiency. The influence of $\delta$-rays below $1.4 \mathrm{GeV}^{\text {nucleon }}{ }^{-1}$ is negligible in this analysis and their effect has no significant difference between isotopes at the same energy, as $M \gg m_{e}$.

\subsubsection{Background}

The $Z=2$ charge separation from $Z<2$ and $Z>2$ samples was studied with MC simulations and inflight data

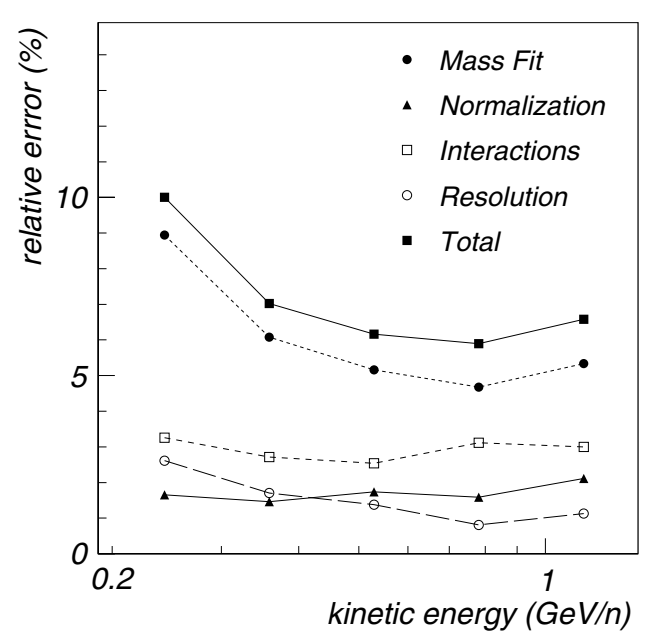

Figure 6. Relative errors on the ${ }^{3} \mathrm{He} /{ }^{4} \mathrm{He}$ ratio measurement as a function of the kinetic energy per nucleon. The total error (filled squares) is obtained from the sum in quadrature of all the other contributions. The lines are to guide the eye.

of $e^{-}, p, \mathrm{He}$, and heavier ions. Proton (ion) beam data at CERN-PS (GSI) provided additional validation at $0.75,2.0$, 3.6 , and $8 \mathrm{GeV}$ nucleon $^{-1}$ of kinetic energy (Alcaraz et al. 1999; Aguilar et al. 2002, 2010). The main potential source of background to the helium sample was protons and deuterons wrongly reconstructed as $Z=2$ particles. Using the single TOF system or the single silicon tracker only, it can be seen, using flight data, that the probability that a $Z=1$ particle is reconstructed as $Z=2$ is below $10^{-3}$, thus affecting the helium sample by $\lesssim 1 \%$. Using the combined measurements obtained from both the detectors, the probability of the wrong charge magnitude was estimated to be $\sim 10^{-7}$ over all energies (Alcaraz et al. 1999). Background from the less abundant $Z>2$ particles is completely negligible compared to the statistical uncertainties in the $\mathrm{He}$ sample (the $\mathrm{He} / \mathrm{Li}$ ratio is $\sim 200$ at the energies considered).

\subsubsection{Energy Losses and Resolution}

Charged CRs that traverse the detector lose energy in the material above the tracking volume. The total energy loss has an appreciable effect on the lowest energy bins. The energy losses by $Z=2$ nuclei were estimated and parameterized with the MC simulation program. We made an event-by-event correction to our data according to the average losses.

Once the above corrections are performed, the relation between the reconstructed energy of detected particles, $E_{\mathrm{REC}}$, and their true energy, $E_{\mathrm{TOI}}$, is still affected by the finite resolution of the measurement. The probability of a bin-to-bin migration $P\left(E_{\mathrm{REC}} \mid E_{\mathrm{TOI}}\right)$ for He was estimated to affect only adjacent energy bins, to be symmetric, and barely isotope dependent. Through these matrix elements, we estimated that the measured ${ }^{3} \mathrm{He} /{ }^{4} \mathrm{He}$ ratio has an uncertainty of $1 \%-3 \%$ due to the resolution.

\subsection{Uncertainty Estimate}

In Figure 6, we summarize the various sources of uncertainty in the measurement of the ${ }^{3} \mathrm{He} /{ }^{4} \mathrm{He}$ ratio. Errors are organized in four categories.

1. Mass fit. The dominant source of uncertainty ( $~ 5 \%-9 \%)$ is that associated with fits to the mass distributions (Section 3.1). The errors were determined directly from 
Table 3

Uncertainty Summary for the Measured Isotopic Ratios

\begin{tabular}{|c|c|c|c|c|c|c|c|c|c|}
\hline \multirow{2}{*}{$\begin{array}{l}\text { Error } \\
\text { Type (Effect) }\end{array}$} & \multicolumn{5}{|c|}{${ }^{3} \mathrm{He} /{ }^{4} \mathrm{He}$ vs. Energy $\left(\mathrm{GeV}\right.$ nucleon $\left.{ }^{-1}\right)$} & \multicolumn{4}{|c|}{ Ratios in $0.2-1.4 \mathrm{GeV}$ nucleon $^{-1}$} \\
\hline & $0.2-0.3$ & $0.4-0.44$ & $0.44-0.64$ & $0.64-0.95$ & $0.95-1.4$ & ${ }^{3} \mathrm{He} /{ }^{4} \mathrm{He}$ & ${ }^{6} \mathrm{Li} /{ }^{7} \mathrm{Li}$ & ${ }^{7} \mathrm{Be} /{ }^{9+10} \mathrm{Be}$ & ${ }^{10} \mathrm{~B} /{ }^{11} \mathrm{~B}$ \\
\hline Mass fit ( $\delta A / A$ and statistics) & $8.9 \%$ & $6.1 \%$ & $5.2 \%$ & $4.7 \%$ & $5.3 \%$ & $4.5 \%$ & $9.0 \%$ & $15.9 \%$ & $12.2 \%$ \\
\hline Normalization $\left(\mathcal{N}-\mathcal{N}_{\mathrm{E}}\right)$ & $1.0 \%$ & $0.9 \%$ & $1.3 \%$ & $1.1 \%$ & $1.9 \%$ & $1.5 \%$ & $2.3 \%$ & $3.0 \%$ & $2.9 \%$ \\
\hline Normalization (acceptance) & $1.3 \%$ & $1.2 \%$ & $1.1 \%$ & $1.1 \%$ & $1.0 \%$ & $0.5 \%$ & $0.5 \%$ & $0.6 \%$ & $0.5 \%$ \\
\hline Interactions (inelastic) & $1.6 \%$ & $1.6 \%$ & $1.8 \%$ & $1.9 \%$ & $2.0 \%$ & $2.0 \%$ & $2.1 \%$ & $2.7 \%$ & $2.4 \%$ \\
\hline Interactions (fragmentation) & $2.8 \%$ & $2.2 \%$ & $1.8 \%$ & $2.5 \%$ & $2.3 \%$ & $2.7 \%$ & $2.4 \%$ & $2.6 \%$ & $2.4 \%$ \\
\hline Total uncertainty & $10.0 \%$ & $7.0 \%$ & $6.2 \%$ & $5.9 \%$ & $6.6 \%$ & $5.8 \%$ & $9.8 \%$ & $16.7 \%$ & $13.0 \%$ \\
\hline
\end{tabular}

Note. The various contributions are described in Section 3.3.

the $1 \sigma$ uncertainties in the $\chi^{2}$ statistics of the fitting procedure. These errors are due to statistical fluctuations of the measured data and the inability of the spectrometer to separate the different masses within the mass resolution $\delta A / A$.

2. Normalization. As discussed in Section 3.1, two parameters $\mathcal{M}$ (ratio) and $\mathcal{N}$ (normalization) were fitted. An ideal fit should lead to $\mathcal{N}$ equal to the number of measured events, $\mathcal{N}_{\mathrm{E}}$. We took the relative difference $\mathcal{N}-\mathcal{N}_{\mathrm{E}}$ as a source of systematic error $(\sim 1 \%)$. Another contribution $(\sim 1 \%)$ is due to the acceptance correction factors estimated with our MC simulation program (Section 3.2.1).

3. Interactions. Our results rely partially on hadronic interaction models, as discussed in Section 3.2.2. Similarly to Wang et al. (2002), we assumed an uncertainty of $10 \%$ in the inelastic cross sections, which corresponds to $2 \%$ of systematic uncertainty. For the fragmentation channel ${ }^{4} \mathrm{He} \rightarrow{ }^{3} \mathrm{He}$, we assumed an uncertainty in the associated cross section equal to that cross section, obtaining an uncertainty of $2 \%-3 \%$ in the measured ${ }^{3} \mathrm{He} /{ }^{4} \mathrm{He}$ ratio. Uncertainties in the material thickness were found to be negligible.

4. Resolution. As discussed in Section 3.2.5, our measurement is affected by the finite energy resolution of the TOF system. A systematic uncertainty of $1 \%-3 \%$ was estimated to account for this effect.

The overall error (filled squares in Figure 6) is taken to be the sum in quadrature of the different contributions. All these uncertainties are also reported in Table 3.

\subsection{Lithium, Beryllium, and Boron}

In our previous work (Aguilar et al. 2010), the lithium isotopic composition was determined between 2.5 and $6.3 \mathrm{GV}$ of magnetic rigidity. Here we present a unified analysis of the lithium, beryllium, and boron isotopes between 0.2 and $1.4 \mathrm{GeV}$ of kinetic energy per nucleon. In this measurement, we followed the same procedure as for the helium analysis. All the steps described in Section 3.1 were repeated for the study of the ratios ${ }^{6} \mathrm{Li} /{ }^{7} \mathrm{Li},{ }^{7} \mathrm{Be} /\left({ }^{9} \mathrm{Be}+{ }^{10} \mathrm{Be}\right)$, and ${ }^{10} \mathrm{~B} /{ }^{11} \mathrm{~B}$. In this section, we outline the essential parts of the $Z>2$ analysis.

The capability of the spectrometer to separate isotopes close in mass was more critical for $Z>2$ and the charge identification capabilities were limited by the use of tracker information only. However, the most limiting factor for the $\mathrm{Li}-\mathrm{Be}-\mathrm{B}$ study was the statistics. Hence, we performed the measurement with just one energy bin from 0.2 to $1.4 \mathrm{GeV}$ nucleon ${ }^{-1}$ and also included data from the Mir-docking (2) and post-docking, non-nadir pointing (3) phases (Section 2). As in our previous work, for
Table 4

Fit Results and Correction Factors for the Ratios ${ }^{3} \mathrm{He} /{ }^{4} \mathrm{He},{ }^{6} \mathrm{Li} /{ }^{7} \mathrm{Li}$, ${ }^{7} \mathrm{Be} /\left({ }^{9} \mathrm{Be}+{ }^{10} \mathrm{Be}\right)$, and ${ }^{10} \mathrm{~B} /{ }^{11} \mathrm{~B}$ in the Range $0.2-1.4 \mathrm{GeV}$ of Kinetic Energy per Nucleon

\begin{tabular}{lcccccc}
\hline \hline Ratio & Events & Fit Results & $\chi^{2} / \mathrm{df}$ & $\delta A / A$ & ACorr & FCorr \\
\hline${ }^{3} \mathrm{He} /{ }^{4} \mathrm{He}$ & 18,035 & $0.174 \pm 0.009$ & $67.7 / 34$ & $12.7 \%$ & 1.02 & 0.98 \\
${ }^{6} \mathrm{Li} /{ }^{7} \mathrm{Li}$ & 1,046 & $0.951 \pm 0.086$ & $16.1 / 23$ & $13.6 \%$ & 0.97 & 0.99 \\
${ }^{7} \mathrm{Be} /{ }^{9+10} \mathrm{Be}$ & 400 & $1.512 \pm 0.238$ & $19.9 / 23$ & $13.8 \%$ & 0.96 & 0.99 \\
${ }^{10} \mathrm{~B} /{ }^{11} \mathrm{~B}$ & 1,598 & $0.494 \pm 0.060$ & $28.0 / 29$ & $13.9 \%$ & 0.96 & 0.99 \\
\hline
\end{tabular}

data collected during phase (2), a geometric cut on the Mir shadow was applied to the acceptance (Aguilar et al. 2010). The geomagnetic regions considered and the event selection criteria were the same as for the helium analysis. Only four hits were required in the tracker, compared to five in the helium analysis. The particle charge was assigned using the identification algorithm described in Aguilar et al. (2010) and Tomassetti (2009) that was specifically optimized for the $Z>2$ species.

Results from the mass composition fit are shown in Figure 7. For comparison, the measurement was also performed on the average ratio of ${ }^{3} \mathrm{He} /{ }^{4} \mathrm{He}$ over this energy range. The large statistical fluctuations of the $Z>2$ data are apparent from the figure, in particular for the less abundant beryllium isotopes (400 events in total). However, the agreement between the measured masses (filled circles) and the simulated histograms (solid lines) was satisfactory. The TOI corrections to the measured composition followed the procedure described in Section 3.2. In contrast to He, the $Z>2$ acceptances were found to be a bit smaller for the heavier isotopes $\left({ }^{7} \mathrm{Li},{ }^{9,10} \mathrm{Be}\right.$, and $\left.{ }^{11} \mathrm{~B}\right)$ than the lighter ones $\left({ }^{6} \mathrm{Li},{ }^{7} \mathrm{Be}\right.$, and $\left.{ }^{10} \mathrm{~B}\right)$, indicating the dominance of nuclear interactions over other effects (see Section 3.2.1). The $Z>2$ mass resolutions, $\delta A / A$, were found to be $\sim 8 \%$ larger than for the $Z=2$ case, reflecting the slight charge dependence of the spectrometer performance in particle tracking and timing. Corrections for fragmentation were also performed, considering the channels ${ }^{7} \mathrm{Li} \rightarrow{ }^{6} \mathrm{Li},{ }^{10,9} \mathrm{Be} \rightarrow{ }^{7} \mathrm{Be}$, and ${ }^{11} \mathrm{~B} \rightarrow{ }^{10} \mathrm{~B}$.

A summary of fit results, TOI corrections, and resolutions is given in Table 4.

The errors were estimated as discussed in Section 3.3. The dominant error is that arising from the $\chi^{2}$ fit procedure: the small $Z>2$ statistics and the broader mass distributions led to less constrained composition fits. Background from helium was estimated not to affect the lithium measurement, as for $\mathrm{He}-\mathrm{Li}$ charge separation the TOF information was still usable (Aguilar et al. 2010). More critical was the contamination in the $Z=4$ sample from adjacent charges that led to larger systematic errors in the beryllium measurement. This channel 

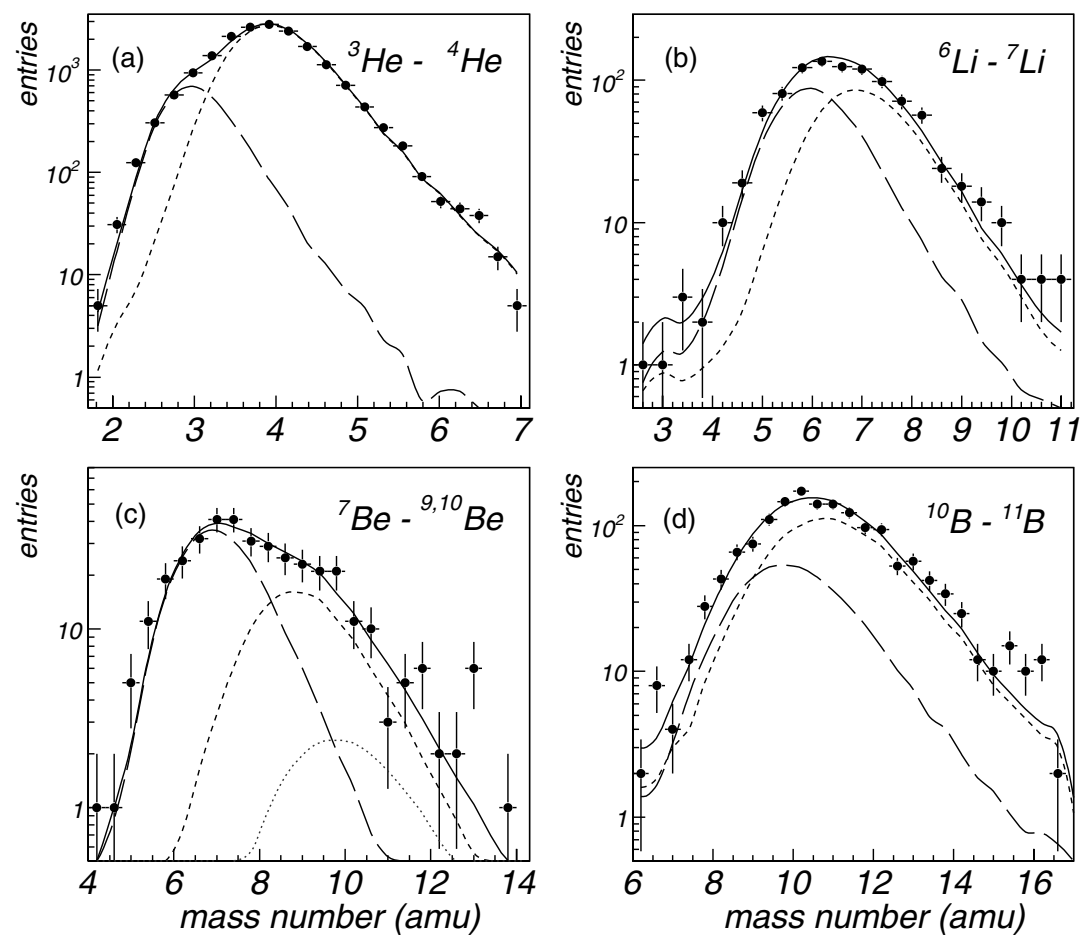

Figure 7. Reconstructed mass distributions of events reconstructed as (a) helium, (b) lithium, (c) beryllium, and (d) boron from data (filled circles) and MC samples of ${ }^{6} \mathrm{Li}-{ }^{7} \mathrm{Li},{ }^{7} \mathrm{Be}-{ }^{9} \mathrm{Be}$, and ${ }^{10} \mathrm{~B}-{ }^{11} \mathrm{~B}$ (lighter isotope: long-dashed lines; heavier isotope: short-dashed lines, sum: solid line). In (c) the dotted line indicates the contribution of ${ }^{10} \mathrm{Be}$, see the text.

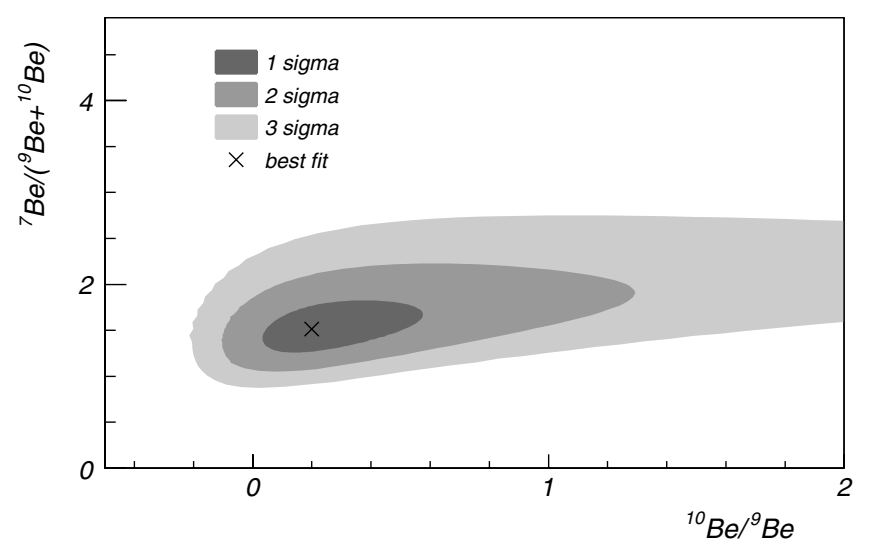

Figure 8. Contour levels of the fitting parameter ${ }^{7} \mathrm{Be} /\left({ }^{9} \mathrm{Be}+{ }^{9} \mathrm{Be}\right)$ and ${ }^{10} \mathrm{Be} /{ }^{9} \mathrm{Be}$ corresponding to $1 \sigma, 2 \sigma$, and $3 \sigma$ bounds of uncertainty in the $\chi^{2}$ statistics.

was also limited by the inability to separate ${ }^{9} \mathrm{Be}$ from ${ }^{10} \mathrm{Be}$. While for the other ratios it can be safely assumed that each charged species is composed of only two long-lived isotopes, a few percent of ${ }^{10} \mathrm{Be}$ has been measured in the $\mathrm{CR}$ flux in addition to the more abundant isotopes ${ }^{7} \mathrm{Be}$ and ${ }^{9} \mathrm{Be}$ (Hams et al. 2004; Webber et al. 2002). We therefore determined the ratio ${ }^{7} \mathrm{Be} /\left({ }^{9} \mathrm{Be}+{ }^{10} \mathrm{Be}\right)$ simultaneously with the additional parameter ${ }^{10} \mathrm{Be} /{ }^{9} \mathrm{Be}$ in our composition fit. The latter was accounted for in the proper determination of the ratio ${ }^{7} \mathrm{Be} /\left({ }^{9} \mathrm{Be}+{ }^{10} \mathrm{Be}\right)$ and its corresponding error. As shown in Figure 8, the ratio ${ }^{10} \mathrm{Be} /{ }^{9} \mathrm{Be}$ is poorly constrained by the data (between $\sim 0$ and $\sim 0.6$ within $1 \sigma$ of uncertainty). Figure 8 also shows that the uncertainty in the ${ }^{10} \mathrm{Be} /{ }^{9} \mathrm{Be}$ ratio has no dramatic consequences on the ${ }^{7} \mathrm{Be} /\left({ }^{9} \mathrm{Be}+{ }^{10} \mathrm{Be}\right)$ ratio, given the weak correlation of the two parameters. The contribution from inelastic collisions and fragmentation was estimated as described in Section 3.2.2.
Similar values as for helium $(\sim 2 \%-3 \%)$ were found for both the effects. Finally, the errors from the TOF energy resolution and from the MC acceptance estimation were smaller than $1 \%$. The total error assigned to the measurements was obtained as the sum in quadrature of all the contributions noted. A detailed summary is provided in Table 3 .

\section{RESULTS AND DISCUSSION}

In the previous sections, we have described the analysis procedure adopted for the determination of the ratios ${ }^{3} \mathrm{He} /{ }^{4} \mathrm{He}$, ${ }^{6} \mathrm{Li} /{ }^{7} \mathrm{Li},{ }^{7} \mathrm{Be} /\left({ }^{9} \mathrm{Be}+{ }^{10} \mathrm{Be}\right)$, and ${ }^{10} \mathrm{~B} /{ }^{11} \mathrm{~B}$. The TOI corrections turned out to be of the same order of magnitude as the estimated uncertainties; hence, the gross features of the measured ratios were apparent directly from the fits to the mass distributions. The error from the fitting procedure was considerably larger than the other contributions. The most important limitations were the mass resolution (for $\mathrm{He}$ ) and the limited statistics (for $\mathrm{Li}-\mathrm{Be}-\mathrm{B}$ ). In particular, the mass resolution was limited by multiple scattering (affecting $\delta R / R$ at $\sim 0.2 \mathrm{GeV}$ nucleon ${ }^{-1}$ ) and the TOF resolution (affecting $\delta \beta / \beta$ at $\sim 1.4 \mathrm{GeV}$ nucleon ${ }^{-1}$ ).

The results with all corrections applied are presented in Table 5. Results for the isotopic ratio ${ }^{3} \mathrm{He} /{ }^{4} \mathrm{He}$ as a function of the kinetic energy per nucleon are shown in Figure 9 between 0.2 and $1.4 \mathrm{GeV}_{\text {nucleon }}{ }^{-1}$ (filled circles). The error bars represent the total errors as discussed in Section 3.3. The figure also shows the existing data between 0.1 and $10 \mathrm{GeV}$ nucleon $^{-1}$ measured by the balloon-borne experiments BESS (Wang et al. 2002), IMAX (Reimer et al. 1998), the first flight of SMILI (Beatty et al. 1993), Hatano et al. (1995), and Webber \& Yushak (1983). Among these, our data are the only data collected directly in space. Our results agree well with data collected by BESS in its first flight in 1993.

Figure 10 shows our results for $\mathrm{Li}-\mathrm{Be}-\mathrm{B}$. The AMS-01 data are compared with measurements made by the space 


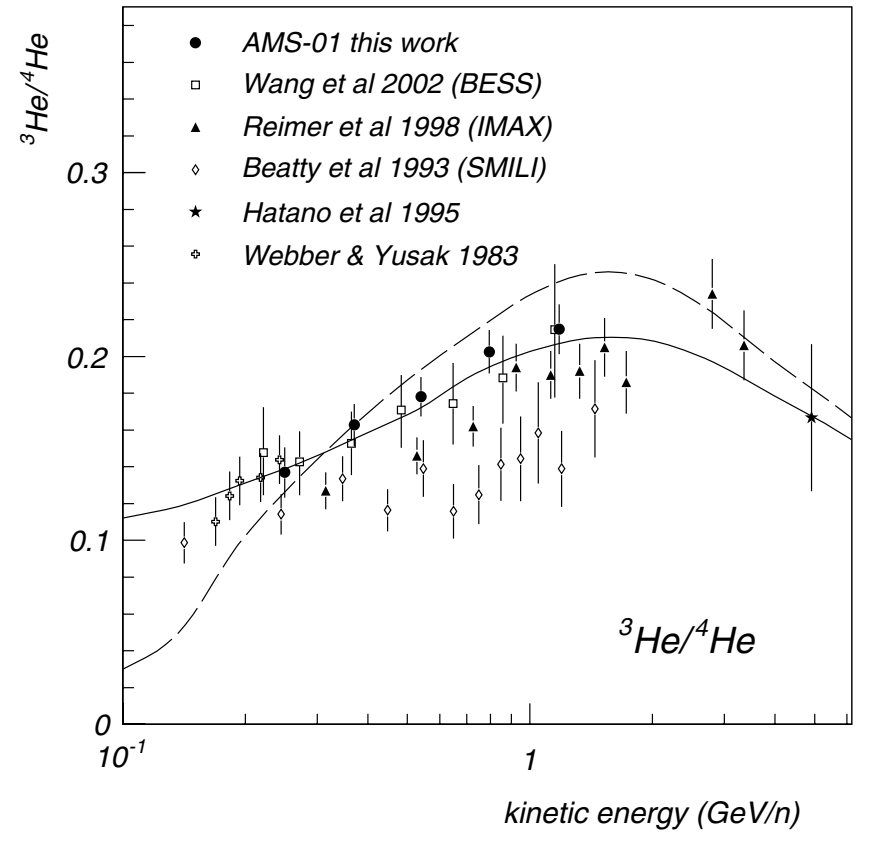

Figure 9. Results for the ratio ${ }^{3} \mathrm{He} /{ }^{4} \mathrm{He}$ between 0.2 and $1.4 \mathrm{GeV}$ nucleon ${ }^{-1}$ of kinetic energy. Other data are from BESS (Wang et al. 2002), IMAX (Reimer et al. 1998), Hatano et al. (1995), SMILI (Beatty et al. 1993), and Webber \& Yushak (1983). The dashed line is the model calculation for the LIS ratio obtained with GALPROP (Strong \& Moskalenko 1998). The modification of this by solar modulation for 1998 June is shown by the solid line.

experiments CRIS on ACE (de Nolfo et al. 2001), Voyager (Webber et al. 2002), Ulysses (Connell 1998), IMP 7/8 (GarciaMunoz 1977), ISEE 3 (Wiedenbeck \& Greiner 1980), and with balloon data from ISOMAX (Hams et al. 2004) and GSFC (Hagen et al. 1977). Our results are consistent with these data within uncertainties, in particular with ISOMAX.

In Figure 11(a), we report the ${ }^{3} \mathrm{He}$ and ${ }^{4} \mathrm{He}$ differential spectra. These spectra are obtained by the combination of the ${ }^{3} \mathrm{He} / \mathrm{He}$ and ${ }^{4} \mathrm{He} / \mathrm{He}$ fractions, given directly by the ${ }^{3} \mathrm{He} /{ }^{4} \mathrm{He}$ ratio, with the AMS-01 helium spectrum previously published in Alcaraz et al. (2000). The ${ }^{3} \mathrm{He}$ and ${ }^{4} \mathrm{He}$ data points and their errors were extracted through a logarithmic interpolation of helium data
Table 5

Results for the Isotopic Ratios and Fluxes at the Top of Instrument

\begin{tabular}{lccc}
\hline \hline Energy $^{\mathrm{a}}$ & ${ }^{3} \mathrm{He} /{ }^{4} \mathrm{He}$ Ratio & ${ }^{3} \mathrm{He}$ Flux & \\
\hline $0.20-0.30$ & $0.137 \pm 0.014$ & $23.3 \pm 2.6$ & $170 \pm 19$ \\
$0.30-0.44$ & $0.163 \pm 0.011$ & $24.7 \pm 2.2$ & $152 \pm 14$ \\
$0.44-0.64$ & $0.178 \pm 0.011$ & $21.6 \pm 1.8$ & $121 \pm 10$ \\
$0.64-0.95$ & $0.203 \pm 0.012$ & $17.5 \pm 1.4$ & $86.5 \pm 6.9$ \\
$0.95-1.40$ & $0.215 \pm 0.014$ & $12.2 \pm 1.0$ & $56.6 \pm 4.7$ \\
\hline Energy ${ }^{\mathrm{a}}$ & ${ }^{2} \mathrm{H} /{ }^{4} \mathrm{He}$ Ratio & Ratios in $0.2-1.4 \mathrm{GeV} \mathrm{nucleon}^{-1}$ \\
\hline $0.20-0.30$ & $0.183 \pm 0.024$ & ${ }^{3} \mathrm{He} /{ }^{4} \mathrm{He}$ & $0.173 \pm 0.010$ \\
$0.30-0.44$ & $0.190 \pm 0.020$ & ${ }^{6} \mathrm{Li} /{ }^{7} \mathrm{Li}$ & $0.912 \pm 0.090$ \\
$0.44-0.64$ & $0.188 \pm 0.021$ & ${ }^{7} \mathrm{Be} /{ }^{9+10} \mathrm{Be}$ & $1.450 \pm 0.242$ \\
$0.64-0.95$ & $0.204 \pm 0.027$ & ${ }^{10} \mathrm{~B} /{ }^{11} \mathrm{~B}$ & $0.4695 \pm 0.061$ \\
\hline
\end{tabular}

Notes.

${ }^{a}$ Kinetic energy is given units of $\mathrm{GeV}$ nucleon ${ }^{-1}$.

${ }^{\mathrm{b}}$ Fluxes are given in units of nucleon $\mathrm{GeV}^{-1} \mathrm{~s}^{-1} \mathrm{~m}^{-2} \mathrm{sr}^{-1}$.

along our energy points. An additional $1 \%$ of error was added due to the interpolation procedure. Similarly, the resulting ${ }^{4} \mathrm{He}$ spectrum has been further combined with the galactic deuteron spectrum published in Aguilar et al. (2002) to extract the ratio between deuterons, ${ }^{2} \mathrm{H}$, and their main progenitors ${ }^{4} \mathrm{He}$. The AMS-01 data analysis of ${ }^{2} \mathrm{H}$ is described in Section 4.6 of Aguilar et al. (2002), where the extraction of the deuteron signal from the vast proton background is discussed quantitatively and the absolute deuteron spectrum is presented in different geomagnetic latitude ranges. The resulting ${ }^{2} \mathrm{H} /{ }^{4} \mathrm{He}$ ratio is shown in Figure 11(b) together with the previous experiments BESS (Wang et al. 2002), IMAX (de Nolfo et al. 2000), and Webber \& Yushak (1983). While all the measurements give larger ${ }^{2} \mathrm{H} /{ }^{4} \mathrm{He}$ ratios than the model predictions (see below) by up to a factor of two, our results again show good agreement with the data from BESS. These data are also reported in Table 5 .

To describe our data, we show in all plots the model calculations of the conventional reacceleration model used in standard methodologies and described extensively elsewhere (Strong \& Moskalenko 1998). Calculations have been made with the package GALPROP-v50.1. ${ }^{45}$

45 http://galprop.stanford.edu
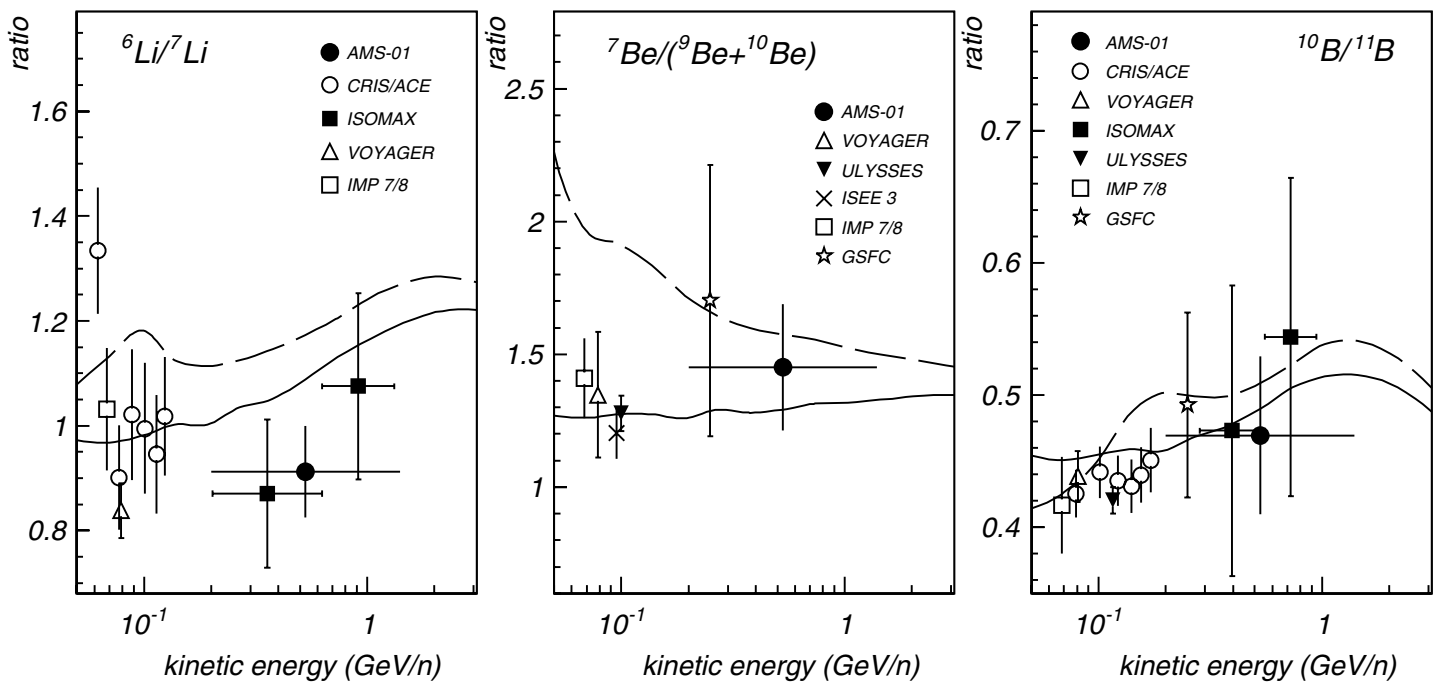

Figure 10. Results for the ratios ${ }^{6} \mathrm{Li} /{ }^{7} \mathrm{Li},{ }^{7} \mathrm{Be} /\left({ }^{9} \mathrm{Be}+{ }^{10} \mathrm{Be}\right)$, and ${ }^{10} \mathrm{~B} /{ }^{11} \mathrm{~B}$ between 0.2 and $1.4 \mathrm{GeV}$ nucleon ${ }^{-1}$ of kinetic energy $(\bullet=\mathrm{AMS}-01$ (present work), $\circ=$ CRIS/ACE (de Nolfo et al. 2001), $\mathbf{\square}=$ ISOMAX (Hams et al. 2004), $\nabla=$ Voyager (Webber et al. 2002), $\boldsymbol{\nabla}=$ Ulysses (Connell 1998), $\times=$ ISEE 3 (Wiedenbeck \& Greiner 1980), $\square=I M P 7 / 8$ (Garcia-Munoz 1977), and $\star=$ GSFC (Hagen et al. 1977)). The dashed (solid) lines are the model calculations for the LIS (solar modulated) ratios obtained with GALPROP (Strong \& Moskalenko 1998). 

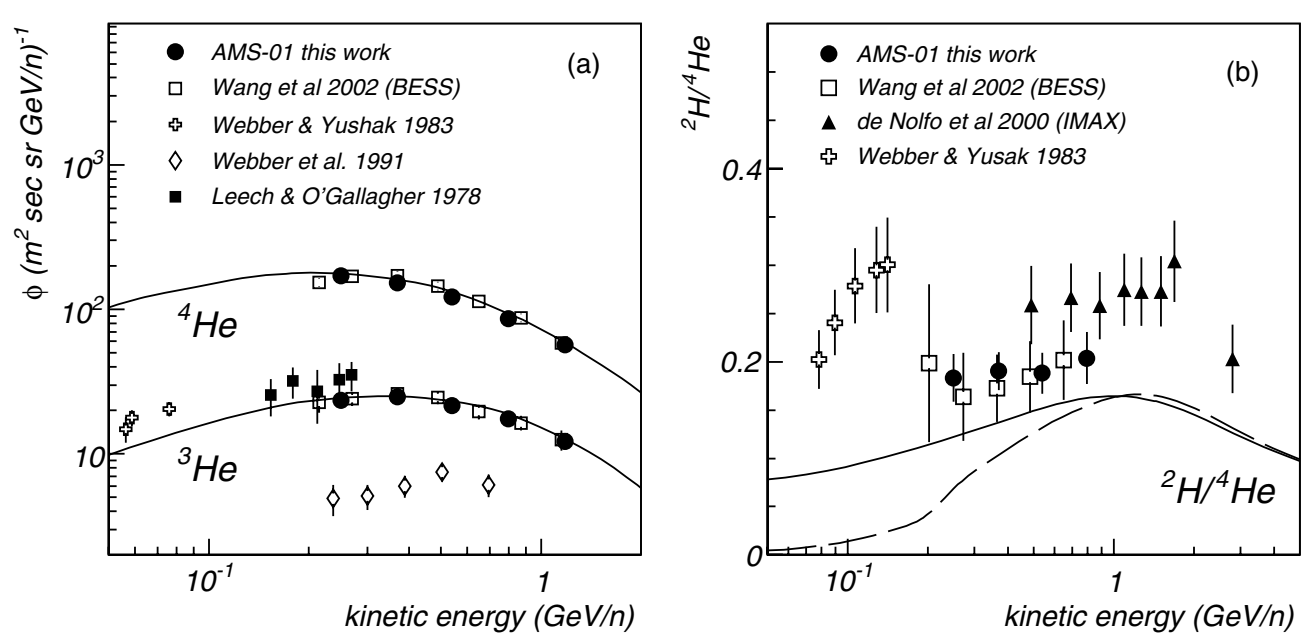

Figure 11. (a) Differential spectra of ${ }^{3} \mathrm{He}$ and ${ }^{4} \mathrm{He}$. Our data have been derived from our earlier published helium spectrum combined with the ${ }^{3} \mathrm{He} /{ }^{4} \mathrm{He}$ ratio of this work. Errors are smaller than the size of the circles. (b) The ratio ${ }^{2} \mathrm{H} /{ }^{4} \mathrm{He}$ derived from our earlier work on ${ }^{2} \mathrm{H}$ flux. In both figures, other data are from balloon-borne experiments (Wang et al. 2002; Webber \& Yushak 1983; Webber et al. 1991; Leech \& O'Gallagher 1978; de Nolfo et al. 2000). The dashed (solid) lines are the model calculations for the LIS (solar modulated) spectra obtained with GALPROP (Strong \& Moskalenko 1998).

Table 6

Propagation Parameter Set

\begin{tabular}{lcc}
\hline \hline Parameter & Name & Value \\
\hline Injection, break value & $R_{B}(\mathrm{GV})$ & 9 \\
Injection, index below $R_{B}$ & $v_{1}$ & 1.80 \\
Injection, index above $R_{B}$ & $v_{2}$ & 2.35 \\
Diffusion, magnitude & $D_{0}\left(\mathrm{~cm}^{2} \mathrm{~s}^{-1}\right)$ & $5 \times 10^{28}$ \\
Diffusion, index & $\delta$ & 0.41 \\
Diffusion, ref. rigidity & $R_{0}(\mathrm{GV})$ & 4 \\
Reacceleration, Alfvén speed & $v_{A}\left(\mathrm{~km} \mathrm{~s}^{-1}\right)$ & 32 \\
Galactic halo, radius & $\mathcal{R}(\mathrm{kpc})$ & 30 \\
Galactic halo, height & $z_{\mathrm{h}}(\mathrm{kpc})$ & 4 \\
Solar modulation parameter & $\phi(\mathrm{MV})$ & 450 \\
\hline
\end{tabular}

GALPROP solves the diffusion-transport equation for a given source distribution and boundary conditions for the galactic CRs, providing steady-state solutions for the local interstellar spectra (LIS) for all the charged CRs up to $Z=28$. The diffusion of CRs through the magnetic halo is described by means of a rigidity-dependent diffusion coefficient $D=\beta D_{0}\left(R / R_{0}\right)^{\delta}$, where $D_{0}$ and $R_{0}$ fix the normalization, and the spectral index $\delta$ drives its rigidity dependence. The reacceleration of charged particles due to scattering on hydromagnetic waves is described as a diffusion in momentum space. This process is controlled by the Alfvén speed of plasma waves moving in the interstellar medium, $v_{A}$. The code also describes energy losses due to ionization or Coulomb scattering, and catastrophic losses over the galactic disk, making use of a large compilation of cross section data and decay rates. To decouple all the transport equations, the fragmentation network starts with the heaviest nucleus and works downward in mass, processing primary and all secondary nuclei produced by the cascade. This loop is repeated twice.

In the parameter setting considered here, no tuning was done to our isotopic data. The nucleon injection spectrum is taken as a "broken" power law in rigidity to better match our total helium and proton spectra from Aguilar et al. (2002). Two indices $v_{1}$ and $v_{2}$ were used below and above $R_{B}$. The cross section database was extended using the updated cross section list from the version v54 (Vladimirov et al. 2011), which includes the production of ${ }^{2} \mathrm{H}$ and ${ }^{3} \mathrm{He}$ from fragmentation of heavier isotopes. The transport parameters $D_{0}, \delta$, and $v_{A}$ are consistent with our B/C ratio from Aguilar et al. (2010). In our description, we used a cylindrically symmetric model of the galactic halo with radius $\mathcal{R}=30 \mathrm{kpc}$ and height $z_{\mathrm{h}}=4 \mathrm{kpc}$. The relevant parameters are reported in Table 6; the remaining specifications are as in the file galdef_50p_599278 provided with the package.

In the ratios of Figures 9-11, local interstellar (dashed lines) and heliospheric propagated (solid lines) calculations are shown. The heliospheric modulation is treated using the force field approximation (Gleeson \& Axford 1968), with $\phi=450 \mathrm{MV}$ as the modulation parameter to characterize the modulation strength for 1998 June. This is also in accordance with the study performed in Wiedenbeck et al. (2009) over the full solar cycle 23 . It should be noted, however, that the force field approximation has no predictive power, i.e., the value employed for the parameter $\phi$ is contextual to the propagation framework adopted to predict the interstellar spectra of the CR elements. For instance, the deuteron flux in Aguilar et al. (2002) was described using $\phi=650 \mathrm{MV}$ after assuming a pure power-law energy spectrum in the ${ }^{2} \mathrm{H}$ LIS.

Though large uncertainties are still present in the heliospheric propagation, the general trend is that higher modulation levels correspond to lower values of the ${ }^{3} \mathrm{He} /{ }^{4} \mathrm{He}$ ratio. AMS-01 and BESS data come from periods of relatively quiet solar activity as do data from Webber \& Yushak ( $\phi \approx 400-650 \mathrm{MV})$. In particular, the periods of 1998 June (AMS-01 flight) and 1993 July (BESS flight) were characterized by very similar solar conditions according to the sunspot data (Temmer et al. 2002) and can be directly compared. Stronger modulations were present when IMAX $(\phi \approx 700-850 \mathrm{MV})$ and SMILI $(\phi \approx 1200-1300 \mathrm{MV})$ were active.

In summary, the secondary to primary ratio ${ }^{3} \mathrm{He} /{ }^{4} \mathrm{He}$, which is much more sensitive to the propagation parameters, seems to be well described by the model under these astrophysical assumptions. The model is also consistent with the $Z>2$ ratios of Figure 10, though large errors are present in the current data. As the $\mathrm{Li}-\mathrm{Be}-\mathrm{B}$ elements are of secondary origin, 
these ratios are less sensitive to the galactic transport and may be useful to investigate the nuclear aspects of the CR propagation (fragmentation, decay, and breakup). Conversely, our ${ }^{2} \mathrm{H} /{ }^{4} \mathrm{He}$ data of Figure 11(b) give a larger ratio than the model predictions, and this tendency is also apparent from the other experiments. Understanding this possible discrepancy may require a thorough investigation of the ${ }^{2} \mathrm{H}$ production cross sections, in particular for the reactions induced by $\mathrm{CR}$ protons and helium nuclei.

\section{CONCLUSIONS}

The AMS-01 detector measured charged CRs during 10 days aboard the Space Shuttle Discovery in 1998 June. Owing to the large number of helium events collected and the absence of atmospheric effects, we have determined precisely the ratio ${ }^{3} \mathrm{He} /{ }^{4} \mathrm{He}$ in the kinetic energy range from 0.2 to $1.4 \mathrm{GeV}$ nucleon ${ }^{-1}$. The average isotopic ratios ${ }^{6} \mathrm{Li} /{ }^{7} \mathrm{Li}$, ${ }^{7} \mathrm{Be} /\left({ }^{9} \mathrm{Be}+{ }^{10} \mathrm{Be}\right)$, and ${ }^{10} \mathrm{~B} /{ }^{12} \mathrm{~B}$ have been measured in the same energy range. The ratio ${ }^{2} \mathrm{H} /{ }^{4} \mathrm{He}$ and the spectra of ${ }^{3} \mathrm{He}$ and ${ }^{4} \mathrm{He}$ are also reported. Our results agree well with the previous data from BESS and ISOMAX and can provide further constraints to the astrophysical parameters of CR propagation. In the analysis procedure adopted in this work, MC simulations were essential for understanding the instrument performance, its acceptance, the role of interactions and for modeling the mass distributions. We expect, with AMS-02, to achieve much more precise results over wider energy ranges.

The support of INFN, Italy, ETH Zurich, the University of Geneva, the Chinese Academy of Sciences, Academia Sinica and National Central University, Taiwan, the RWTH Aachen, Germany, the University of Turku, the University of Technology of Helsinki, Finland, the US DOE and MIT, CIEMAT, Spain, LIP, Portugal and IN2P3, France is gratefully acknowledged. The success of the first AMS mission is due to many individuals and organizations outside of the collaboration. The support of NASA was vital in the inception, development, and operation of the experiment. Support from the Max-Planck Institute for Extraterrestrial Physics, from the space agencies of Germany (DLR), Italy (ASI), France (CNES), and China and from
CSIST, Taiwan also played important roles in the success of AMS.

\section{REFERENCES}

Aguilar, M., et al. 2002, Phys. Rep., 366, 331 (AMS-01)

Aguilar, M., et al. 2007, Phys. Lett. B, 646, 145 (AMS-01)

Aguilar, M., et al. 2010, ApJ, 724, 329 (AMS-01)

Ahlen, S. P., et al. 2000, ApJ, 534, 757 (SMILI-II)

Alcaraz, J., et al. 1999, Phys. Lett. B, 461, 387 (AMS-01)

Alcaraz, J., et al. 2000, Phys. Lett. B, 494, 193 (AMS-01)

Beatty, J. J., et al. 1993, ApJ, 413, 268 (SMILI-I)

Brun, R., et al. 1987, DD/EE/84-1, CERN (GEANT3)

Connell, J. J. 1998, ApJ, 501, L59

de Nolfo, G. A., et al. 2000, in AIP Conf. Proc. 528, Proc. ACE2000 Symp., ed. R. A. Mewaldt et al. (Melville, NY: AIP), 425 (CRIS)

de Nolfo, G. A., et al. 2001, Proc. 27th ICRC, Hamburg, Vol. 2, ed. M. Simon, E. Lorenz, \& M. Pohl (Katlenburg-Lindau, Germany: Copernicus), 1667 (CRIS)

Garcia-Munoz, M., Mason, G. M., \& Simpson, J. A. 1977, ApJ, 217, 859 (IMP 7/8)

Gleeson, L. J., \& Axford, W. I 1968, ApJ, 154, 1011

Hagen, F. A., Fisher, A. J., \& Ormes, J. F. 1977, ApJ, 212, 262 (GSFC)

Hams, T., et al. 2004, ApJ, 611, 892 (ISOMAX)

Hatano, Y., Fukada, Y., Saito, Y., Oda, H., \& Yanagita, T. 1995, Phys. Rev. D, 52,6219

Leech, H. W., \& O'Gallagher, J. J. 1978, ApJ, 221, 1110

Moskalenko, I. V., Strong, A. W., Mashnik, S. G., \& Jones, F. C. 2003, Proc. 28th ICRC, Tsukuba, Vol. 2, ed. T. Kajita, Y. Asaoka, A. Kawachi, Y. Matsubara, \& M. Sasaki (Tokyo, Japan: Universal Academy Press), 1917 (arXiv:astro-ph/0306349)

Reimer, O., et al. 1998, ApJ, 496, 490 (IMAX)

Seo, E. S., et al. 1997, Adv. Space Res., 19, 751 (BESS)

Sorge, H. 1995, Phys. Rev. C, 52, 3291 (RQMD)

Strong, A. W., \& Moskalenko, I. V. 1998, ApJ, 509, 212 (GALPROP)

Strong, A. W., Moskalenko, I. V., \& Ptuskin, V. S. 2007, Annu. Rev. Nucl. Part. Syst., 57, 285

Temmer, M., Veronig, A., \& Hanslmeier, A. 2002, A\&A, 390, 707

Tomassetti, N. 2009, PhD thesis, Univ. Perugia, Italy

Vladimirov, A. E., et al. 2011, Comput. Phys. Commun., 182, 1156

Wang, J. Z., et al. 2002, ApJ, 564, 244 (BESS)

Webber, W. R. 1997, Adv. Space Res., 19, 755

Webber, W. R., Golden, R. L., Stochaj, S. J., Ormes, J. F., \& Strittmatter, R. E. 1991, ApJ, 380, 230

Webber, W. R., Lukasiak, A., \& McDonald, F. B. 2002, ApJ, 568, 210 (Voyager)

Webber, W. R., \& Yushak, S. M. 1983, ApJ, 275, 391

Wiedenbeck, M. E., \& Greiner, D. E. 1980, ApJ, 239, L139 (ISEE 3)

Wiedenbeck, M. E., et al. 2009, Proc. 31th ICRC, Łódź, Vol. 1 (Łódź, Poland: Univ. Łódź), 4 